\title{
Exploring $B_{d, s} \rightarrow K K$ decays through flavour symmetries and QCD-factorisation
}

\author{
Sébastien Descotes-Genon ${ }^{a}$, Joaquim Matias $^{b}$ and Javier Virto ${ }^{b}$ \\ ${ }^{a}$ Laboratoire de Physique Théorique, CNRS/Univ. Paris-Sud 11 (UMR 8627), 91405 Orsay Cedex, France \\ ${ }^{b}$ IFAE, Universitat Autònoma de Barcelona, 08193 Bellaterra, Barcelona, Spain
}

(Dated: August 30, 2018)

\begin{abstract}
We present a new analysis of $B_{d, s} \rightarrow K K$ modes within the SM, relating them in a controlled way through $S U(3)$-flavour symmetry and QCD-improved factorisation. We propose a set of sum rules for $B_{d, s} \rightarrow K^{0} \bar{K}^{0}$ observables. We determine $B_{s} \rightarrow K K$ branching ratios and CP-asymmetries as functions of $A_{d i r}\left(B_{d} \rightarrow K^{0} \bar{K}^{0}\right)$, pointing out a conflict between $B R\left(B_{s} \rightarrow K^{+} K^{-}\right)$in the SM and data. Finally, we predict the amount of $U$-spin breaking between $B_{d} \rightarrow \pi^{+} \pi^{-}$and $B_{s} \rightarrow K^{+} K^{-}$.
\end{abstract}

$B_{s}$ decays offer promising prospects in searches for New Physics (NP). But to disentangle NP, it is essential to find new strategies to reduce hadronic uncertainties to obtain precise predictions within the Standard Model (SM). In particular, an ongoing effort has been devoted to $B_{s} \rightarrow K K$ decays within several approaches, mainly QCD factorisation (QCDF) 1, 2] (with its SCET extension [3]) and flavour symmetries [4, 5, 6, 7]. The former is a systematic expansion in $1 / m_{b}$ but has difficulties with phenomenology due to power-suppressed hadronic effects, such as final-state interactions. The latter takes hadronic effects into account but may be affected by large corrections, up to $30 \%$ for $S U(3)$ relations. In this paper, we combine the best of each method to derive SM relations between $B_{d} \rightarrow K^{0} \bar{K}^{0}$ and $B_{s} \rightarrow K K$. We use data when available and exploit flavour symmetries and QCDF when they can be controlled efficiently.

This Letter is organized in the following way. First, we present two new SM relations for the $B_{d, s} \rightarrow K^{0} \bar{K}^{0}$ decays that link the difference between tree and penguin contributions (a well-controlled quantity within QCDF) with observables measured in $B$-experiments. Then we show that flavour symmetry yields interesting relations between hadronic parameters in $B_{d} \rightarrow K^{0} \bar{K}^{0}, B_{s} \rightarrow$ $K^{0} \bar{K}^{0}$, and $B_{s} \rightarrow K^{+} K^{-}$, providing a complementary strategy to $B_{d} \rightarrow \pi^{+} \pi^{-}$[4, 5, 6, 7]. To exploit these relations, we propose to determine the $B_{d} \rightarrow K^{0} \bar{K}^{0}$ hadronic parameters up to a two-fold ambiguity from the branching ratio, the direct $\mathrm{CP}$-asymmetry and the tree-penguin difference. Third, we provide SM predictions for $B_{s} \rightarrow K K$ using this new strategy. Finally, we assess $U$-spin breaking between $B_{s} \rightarrow K^{+} K^{-}$and $B_{d} \rightarrow \pi^{+} \pi^{-}$, of interest for both QCDF and flavoursymmetry approaches.

The SM amplitude for a $B$ decaying into two mesons can be split into tree and penguin contributions [8]:

$$
\bar{A} \equiv A\left(\bar{B}_{q} \rightarrow M \bar{M}\right)=\lambda_{u}^{(q)} T_{M}^{q C}+\lambda_{c}^{(q)} P_{M}^{q C},
$$

with $C$ denoting the charge of the decay products, and the products of CKM factors $\lambda_{p}^{(q)}=V_{p b} V_{p q}^{*}$. Using QCDF 1, 2], one can perform a $1 / m_{b}$-expansion of the amplitude, which gets two kinds of contributions [9]: a factorisable part which can be improved within QCDF and a non-factorisable one from $1 / m_{b}$-suppressed corrections, much more delicate to evaluate.

The tree and penguin contributions in $\bar{B}_{s} \rightarrow K^{+} K^{-}$ and $\bar{B}_{s} \rightarrow K^{0} \bar{K}^{0}$ in QCDF are, respectively:

$$
\begin{aligned}
\hat{T}^{s \pm}= & \bar{\alpha}_{1}+\bar{\beta}_{1} \\
& +\bar{\alpha}_{4}^{u}+\bar{\alpha}_{4 E W}^{u}+\bar{\beta}_{3}^{u}+2 \bar{\beta}_{4}^{u}-\frac{1}{2} \bar{\beta}_{3 E W}^{u}+\frac{1}{2} \bar{\beta}_{4 E W}^{u} \\
\hat{P}^{s \pm}= & \bar{\alpha}_{4}^{c}+\bar{\alpha}_{4 E W}^{c}+\bar{\beta}_{3}^{c}+2 \bar{\beta}_{4}^{c}-\frac{1}{2} \bar{\beta}_{3 E W}^{c}+\frac{1}{2} \bar{\beta}_{4 E W}^{c}(3) \\
\hat{T}^{s 0}= & \bar{\alpha}_{4}^{u}-\frac{1}{2} \bar{\alpha}_{4 E W}^{u}+\bar{\beta}_{3}^{u}+2 \bar{\beta}_{4}^{u}-\frac{1}{2} \bar{\beta}_{3 E W}^{u}-\bar{\beta}_{4 E W}^{u}(4) \\
\hat{P}^{s 0}= & \bar{\alpha}_{4}^{c}-\frac{1}{2} \bar{\alpha}_{4 E W}^{c}+\bar{\beta}_{3}^{c}+2 \bar{\beta}_{4}^{c}-\frac{1}{2} \bar{\beta}_{3 E W}^{c}-\bar{\beta}_{4 E W}^{c}(5)
\end{aligned}
$$

where $\hat{P}^{s C}=P^{s C} / A_{K K}^{s}, \hat{T}^{s C}=T^{s C} / A_{K K}^{s}$ and $A_{K K}^{q}=$ $M_{B_{q}}^{2} F_{0}^{\bar{B}_{q} \rightarrow K}(0) f_{K} G_{F} / \sqrt{2}$. The superscripts identify the channel and the bar denotes quantities for decays with a spectator $s$-quark. The tree and penguin contributions $T^{d 0}$ and $P^{d 0}$ for $\bar{B}_{d} \rightarrow K^{0} \bar{K}^{0}$ have the same structure as eqs. (4) and (5), with unbarred $\alpha$ 's and $\beta$ 's recalling the different nature of the spectator $d$-quark.

At NLO in $\alpha_{s}, \alpha$ 's are linear combinations of vertex corrections, hard-spectator terms and penguin contractions, whereas $\beta$ 's are sums of annihilation contributions. The weights of the various contributions are expressed in terms of $\alpha_{s}$ and Wilson coefficients [2]. The explicit form of the $\bar{\alpha}_{i}^{p}-\alpha_{i}^{p}$ required for the discussion is shown in Sec.II. $\alpha$ 's and $\beta$ 's contain the two most significant terms in the $1 / m_{b}$ expansion: the LO terms, dominated by short distances, and the NLO terms in $1 / m_{b}$ that include the potentially large long-distance corrections. The latter, parameterised in QCDF through quantities denoted $X_{H}$ (in power-corrections to the hard-scattering part of $\alpha_{i}$ ) and $X_{A}$ (in the annihilation parameters $\beta_{i}$ ), are singled out since they may upset the quick convergence of the $1 / m_{b}$ expansion. The other $1 / m_{b}$-suppressed contributions, dominated by short distances, are under control and small, i.e, leading to a $\mathcal{O}(5-10 \%)$ error.

In this Letter, we show that comparing $B_{d^{-}}$and $B_{s^{-}}$ decays into the same final states helps to cancel the potentially large long-distance $1 / m_{b}$-suppressed effects $\left(X_{A, H}\right)$, yielding improved SM predictions. 


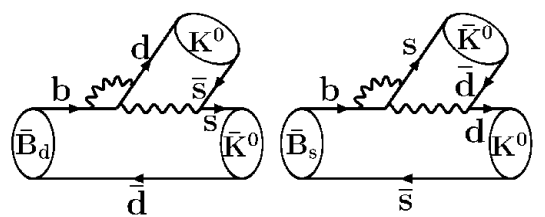

FIG. 1: Diagrams contributing to $\bar{B}_{d} \rightarrow K^{0} \bar{K}^{0}$ (left) and $\bar{B}_{s} \rightarrow K^{0} \bar{K}^{0}$ (right) related through $U$-spin transformations.

I. Sum rules. The difference $\Delta_{d} \equiv T^{d 0}-P^{d 0}$ plays a fundamental role here, since it is free from the troublesome NLO infrared-divergence (modelled by $X_{A, H}$ ) that may be enhanced numerically by the chiral factor $r_{\chi}^{K}=2 m_{K}^{2} / m_{b} / m_{s}$ from twist-3 distribution amplitudes. Hard-scattering $\left(X_{H}\right)$ and annihilation $\left(X_{A}\right)$ terms occur in both penguin and tree contributions, but remarkably they cancel in the short-distance difference:

$$
\begin{aligned}
\Delta_{d} & =A_{K K}^{d}\left[\alpha_{4}^{u}-\alpha_{4}^{c}+\beta_{3}^{u}-\beta_{3}^{c}+2 \beta_{4}^{u}-2 \beta_{4}^{c}\right] \\
& =A_{K K}^{d} \alpha_{s} C_{F} C_{1}\left[\bar{G}\left(m_{c}^{2} / m_{b}^{2}\right)-\bar{G}(0)\right] /\left(4 \pi N_{c}\right),
\end{aligned}
$$

neglecting (small) electroweak contributions. The function $\bar{G}=G_{K}+r_{\chi}^{K} \hat{G}_{K}$ combines one-loop integrals from the penguin terms $P_{4}$ and $P_{6}$ defined in Sec 2.4 in ref. [2]. The same cancellation of long-distance $1 / m_{b}$-corrections happens for $\Delta_{s} \equiv T^{s 0}-P^{s 0}$. Taking into account the uncertainties coming from the QCDF inputs [2], we get $\Delta_{d}=(1.09 \pm 0.43) \cdot 10^{-7}+i(-3.02 \pm 0.97) \cdot 10^{-7} \mathrm{GeV}$ and $\Delta_{s}=(1.03 \pm 0.41) \cdot 10^{-7}+i(-2.85 \pm 0.93) \cdot 10^{-7} \mathrm{GeV}$.

These two theoretical quantities can be related to observables, namely the corresponding branching ratio and coefficients of the time-dependent CP-asymmetry:

$$
\begin{aligned}
\frac{\Gamma\left(B_{d}(t)\right.}{\Gamma\left(B_{d}(t)\right.} & \left.\left.\rightarrow K^{0} \bar{K}^{0}\right)-\Gamma\left(K^{0} \bar{K}^{0}\right)+\Gamma(t) \rightarrow K^{0} \bar{K}^{0}(t) \rightarrow K^{0} \bar{K}^{0}\right) \\
& =\frac{A_{d i r}^{d 0} \cos (\Delta M \cdot t)+A_{m i x}^{d 0} \sin (\Delta M \cdot t)}{\cosh \left(\Delta \Gamma_{d} t / 2\right)-A_{\Delta}^{d 0} \sinh \left(\Delta \Gamma_{d} t / 2\right)}
\end{aligned}
$$

where we define [4]: $A_{\text {dir }}^{d 0}=\left(|A|^{2}-|\bar{A}|^{2}\right) /\left(|A|^{2}+|\bar{A}|^{2}\right)$, $A_{\Delta}^{d 0}+i A_{m i x}^{d 0}=-\left(2 e^{-i \phi_{d}} A^{*} \bar{A}\right) /\left(|A|^{2}+|\bar{A}|^{2}\right)$ and $\phi_{d}$ the phase of $B_{d}-\bar{B}_{d}$ mixing. $A_{\Delta}^{d 0}$ is unlikely to be measured due to the small width difference $\Delta \Gamma_{d}$, but it can be obtained from the other asymmetries by means of the relation $\left|A_{\Delta}^{d 0}\right|^{2}+\left|A_{d i r}^{d 0}\right|^{2}+\left|A_{\text {mix }}^{d 0}\right|^{2}=1$.

One can derive the following relation for $B_{d} \rightarrow K^{0} \bar{K}^{0}$ :

$$
\begin{aligned}
&\left|\Delta_{d}\right|^{2}=\frac{B R^{d 0}}{L_{d}}\left\{x_{1}+[\right.\left.x_{2} \sin \phi_{d}-x_{3} \cos \phi_{d}\right] A_{m i x}^{d 0} \\
&\left.-\left[x_{2} \cos \phi_{d}+x_{3} \sin \phi_{d}\right] A_{\Delta}^{d 0}\right\},
\end{aligned}
$$

where $L_{d}=\tau_{d} \sqrt{M_{B d}^{2}-4 M_{K}^{2}} /\left(32 \pi M_{B d}^{2}\right)$ and:

$$
\begin{aligned}
& x_{1}=\left[\left|\lambda_{c}^{(d)}\right|^{2}+\left|\lambda_{u}^{(d)}\right|^{2}-2\left|\lambda_{c}^{(d)}\right|\left|\lambda_{u}^{(d)}\right| \cos \gamma\right] / n^{2}, \\
& x_{2}=-\left[\left|\lambda_{c}^{(d)}\right|^{2}+\left|\lambda_{u}^{(d)}\right|^{2} \cos 2 \gamma-2\left|\lambda_{c}^{(d)}\right|\left|\lambda_{u}^{(d)}\right| \cos \gamma\right] / n^{2}, \\
& x_{3}=-\left[1-\cos \gamma \times\left|\lambda_{u}^{(d)}\right| /\left|\lambda_{c}^{(d)}\right|\right] / n,
\end{aligned}
$$

with $n=2\left|\lambda_{c}^{(d)}\right|\left|\lambda_{u}^{(d)}\right| \sin \gamma$. A similar relation between $\Delta_{s}$ and $B_{s} \rightarrow K^{0} \bar{K}^{0}$ observables is obtained by replacing $\left|\lambda_{u}^{(d)}\right| \rightarrow\left|\lambda_{u}^{(s)}\right|,\left|\lambda_{c}^{(d)}\right| \rightarrow-\left|\lambda_{c}^{(s)}\right|$, and $d \rightarrow s$ for all indices.

These sum rules can be used either as a SM consistency test between $B R^{s 0},\left|A_{d i r}^{s 0}\right|$ and $A_{m i x}^{s 0}$ (and similarly for the $B_{d}^{0} \rightarrow K^{0} \bar{K}^{0}$ observables), or as a way to extract the SM value of one observable (say $\left|A_{d i r}^{s 0}\right|$ ) in terms of the two others $\left(B R^{s 0}\right.$ and $\left.A_{m i x}^{s 0}\right)$ and $\Delta_{s}$. These relations are free from the long-distance power-suppressed modeldependent quantities $X_{A}$ and $X_{H}$ that are a main error source in the direct computation of $A_{d i r}^{s 0}$ within QCDF.

II. Flavour symmetries and QCDF. Using $U$-spin symmetry, we can relate the two penguin-mediated decays $\bar{B}_{d} \rightarrow K_{0} \bar{K}_{0}$ and $\bar{B}_{s} \rightarrow K_{0} \bar{K}_{0}$, as exemplified in fig. 10 (see also ref. 10] in relation to $B \rightarrow \pi \pi$ ). Let us stress that we work with the operators of the effective Hamiltonian: internal loops have already been integrated out to yield four-quark operators, so that the internal loop of the $u$-penguin is not affected by $U$-spin rotations. $U$-spin breaking should be much smaller here than usual: it does not affect final-state interaction since both decays involve the same outgoing state, and it shows up mainly in power-suppressed effects. This is confirmed by QCDF:

$$
\begin{array}{r}
P^{s 0}=f P^{d 0}\left[1+\left(A_{K K}^{d} / P^{d 0}\right)\left\{\delta \alpha_{4}^{c}-\delta \alpha_{4 E W}^{c} / 2\right.\right. \\
\left.\left.+\delta \beta_{3}^{c}+2 \delta \beta_{4}^{c}-\delta \beta_{3 E W}^{c} / 2-\delta \beta_{4 E W}^{c}\right\}\right], \\
T^{s 0}=f T^{d 0}\left[1+\left(A_{K K}^{d} / T^{d 0}\right)\left\{\delta \alpha_{4}^{u}-\delta \alpha_{4 E W}^{u} / 2\right.\right. \\
\left.\left.+\delta \beta_{3}^{u}+2 \delta \beta_{4}^{u}-\delta \beta_{3 E W}^{u} / 2-\delta \beta_{4 E W}^{u}\right\}\right],
\end{array}
$$

where we define the $U$-spin breaking differences $\delta \alpha_{i}^{p} \equiv$ $\bar{\alpha}_{i}^{p}-\alpha_{i}^{p}$ (id. for $\beta$ ). Apart from the factorisable ratio :

$$
f=A_{K K}^{s} / A_{K K}^{d}=M_{B_{s}}^{2} F_{0}^{\bar{B}_{s} \rightarrow K}(0) /\left[M_{B_{d}}^{2} F_{0}^{\bar{B}_{d} \rightarrow K}(0)\right]
$$

which should be computed on the lattice, $U$-spin breaking arises through $1 / m_{b}$-suppressed contributions in which most long-distance contributions have cancelled out.

First, the hard-spectator scattering $(\delta \alpha)$ probes the difference between $B_{d^{-}}$and $B_{s}$-distribution amplitudes:

$$
\begin{aligned}
\delta \alpha_{4}^{p} & =\alpha_{s} C_{F} C_{3} \pi / N_{c}^{2} \times \delta \lambda_{B} \times\left[\langle\bar{x}\rangle_{K}^{2}+r_{\chi}^{K}\langle x\rangle_{K} X_{H}^{K}\right], \\
\delta \lambda_{B} & =B_{K K}^{s} M_{B_{s}} /\left(A_{K K}^{s} \lambda_{B_{s}}\right)-B_{K K}^{d} M_{B_{d}} /\left(A_{K K}^{d} \lambda_{B_{d}}\right)
\end{aligned}
$$

$B_{K K}^{q}=f_{B_{q}} f_{K}^{2} G_{F} / \sqrt{2},\langle\bar{x}\rangle_{K}$ and $M_{B_{q}} / \lambda_{B_{q}}$ are first and first inverse moments of $K$ and $B_{q}$ distribution amplitudes [2], respectively. $\delta \lambda_{B}$ is expected small, since the dynamics of the heavy-light meson in the limit $m_{b} \rightarrow \infty$ should vary little from $B_{d}$ and $B_{s}$. Second, the annihilation contributions $(\delta \beta)$ contain a $U$-spin breaking part when the gluon is emitted from the light quark in the $B_{d, s}$-meson (this effect from $A_{1}^{i}$ and $A_{2}^{i}$ defined in [2] is neglected in the QCDF model for annihilation terms). 
Taking the hadronic parameters in [2], we obtain $\left|P^{s 0} /\left(f P^{d 0}\right)-1\right| \leq 3 \%$ and $\left|T^{s 0} /\left(f T^{d 0}\right)-1\right| \leq 3 \%$. These relations yield also the constraint $\Delta_{s}=f \Delta_{d}$ up to $1 / m_{b^{-}}$ suppressed corrections, which relates observables in both decays through eq. (8) and its counterpart for $\Delta_{s}$.

Relations exist between $\bar{B}_{d} \rightarrow K_{0} \bar{K}_{0}$ and $\bar{B}_{s} \rightarrow$ $K^{+} K^{-}$as well. A combination of $U$-spin and isospin rotations leads from the penguin contribution in $\bar{B}_{d} \rightarrow$ $K_{0} \bar{K}_{0}$ to that in $\bar{B}_{s} \rightarrow K_{0} \bar{K}_{0}$, then to $\bar{B}_{s} \rightarrow K^{+} K^{-}$, up to electroweak corrections (it corresponds to fig. 11 up to replacing $d \rightarrow u$ in the right-hand diagram). On the other hand, there are no such relations between tree contributions, since $\bar{B}_{s} \rightarrow K^{+} K^{-}$contains tree contributions which have no counterpart in the penguin-mediated decay $\bar{B}_{d} \rightarrow K_{0} \bar{K}_{0}$. This is seen in QCDF as well:

$$
\begin{aligned}
& P^{s \pm}=f P^{d 0}\left[1+\frac{A_{K K}^{d}}{P^{d 0}}\left\{\frac{3}{2}\left(\alpha_{4 E W}^{c}+\beta_{4 E W}^{c}\right)+\delta \alpha_{4}^{c}\right.\right. \\
& \left.\left.+\delta \alpha_{4 E W}^{c}+\delta \beta_{3}^{c}+2 \delta \beta_{4}^{c}-\frac{1}{2}\left(\delta \beta_{3 E W}^{c}-\delta \beta_{4 E W}^{c}\right)\right\}\right], \quad \text { (10) } \\
& \frac{T^{s \pm}}{A_{K K}^{s} \bar{\alpha}_{1}}=1+\frac{T^{d 0}}{A_{K K}^{d} \bar{\alpha}_{1}}+\frac{1}{\bar{\alpha}_{1}}\left\{\bar{\beta}_{1}+\frac{3}{2}\left(\alpha_{4 E W}^{u}+\beta_{4 E W}^{u}\right)\right. \\
& \left.+\delta \alpha_{4}^{u}+\delta \alpha_{4 E W}^{u}+\delta \beta_{3}^{u}+2 \delta \beta_{4}^{u}-\frac{1}{2}\left(\delta \beta_{3 E W}^{u}-\delta \beta_{4 E W}^{u}\right)\right\} .
\end{aligned}
$$

Terms are ordered in decreasing size (in particular, curly brackets in $T^{s \pm}$ should be tiny). From QCDF, we obtain the following bounds: $\left|P^{s \pm} /\left(f P^{d 0}\right)-1\right| \leq 2 \%$ and $\left|T^{s \pm} /\left(A_{K K}^{s} \bar{\alpha}_{1}\right)-1-T^{d 0} /\left(A_{K K}^{d} \bar{\alpha}_{1}\right)\right| \leq 4 \%$. The latter shows that flavour-symmetry breaking corrections are smaller than $T^{d 0} /\left(A_{K K}^{d} \bar{\alpha}_{1}\right)=O(10 \%)$. Fortunately, $T^{s \pm}$ is strongly CKM suppressed in $B_{s} \rightarrow K^{+} K^{-}$so that the uncertainty on its QCDF determination will affect the branching ratio and $\mathrm{CP}$-asymmetries only marginally.

Finally, these relations between $B_{d}$ and $B_{s}$ hadronic parameters are affected by electroweak penguins, small in the SM but potentially enhanced by NP effects.

III. Hadronic parameters in $B_{d} \rightarrow K^{0} \bar{K}^{0}$. The dynamics of $B_{d} \rightarrow K^{0} \bar{K}^{0}$ involves three hadronic real parameters (modulus of the tree, modulus of the penguin and relative phase) which we can pin down through three observables: $B R^{d 0}, A_{d i r}^{d 0}$ and $A_{m i x}^{d 0}$. Only $B R^{d 0}=$ $(0.96 \pm 0.25) \cdot 10^{-6}[1]$ has been measured. However the direct asymmetry $A_{d i r}^{d 0}$ should be observable fairly easily (for instance, $A_{d i r}^{d 0}=0.19 \pm 0.06$ in QCDF) whereas the mixed asymmetry is likely small $\left(A_{m i x}^{d 0}=0.05 \pm 0.05\right.$ in QCDF). If only $A_{d i r}^{d 0}$ becomes available, we have only 2 experimental constraints for 3 hadronic parameters. Then we may exploit a theoretically well-controlled QCDF constraint to get $T^{d 0}$ and $P^{d 0}$ from $B R^{d 0}, A_{d i r}^{d 0}$ and the QCDF value of $\Delta_{d} \equiv T^{d 0}-P^{d 0}$, free from infrared divergences and thus with little model dependence.

This system yields two constraints in the complex plane $\left(x_{P}, y_{P}\right)$ for $P^{d 0}$. First, the branching ratio defining $\rho_{0}^{2} \equiv B R^{d 0} /\left(2 L_{d}\right)$ and the QCDF constraint on $\Delta_{d}$ yield a circular ring of centre $\left(x_{C}, y_{C}\right)$ and radius $r$ :

$$
\begin{aligned}
x_{C}+i y_{C} & =-\Delta_{d}(1-\cos \gamma / R) / a, \\
r^{2} & =\rho_{0}^{2} /\left[a\left|\lambda_{u}^{(d)}\right|^{2}\right]-\left[\sin \gamma\left|\Delta_{d}\right| /(a R)\right]^{2},
\end{aligned}
$$

with $a=1-2 \cos \gamma / R+1 / R^{2}$ and $R=\left|\lambda_{u}^{(d)} / \lambda_{c}^{(d)}\right|$.

The second constraint combines $\Delta_{d}=x_{\Delta_{d}}+i y_{\Delta_{d}}$ and the direct $\mathrm{CP}$ asymmetry $A_{d i r}^{d 0}$ into a diagonal strip:

$$
y_{P} x_{\Delta_{d}}=y_{\Delta_{d}} x_{P}-\rho_{0}^{2} A_{d i r}^{d 0} /\left(2\left|\lambda_{u}^{(d)} \lambda_{c}^{(d)}\right| \sin \gamma\right) .
$$

Numerically, only $\left|A_{\text {dir }}^{d 0}\right|<0.2$ is compatible with both constraints, which intersect in two points with opposite signs for $\operatorname{Im} P^{d 0}$, yielding two solutions for $\left(P^{d 0}, T^{d 0}\right)$.

IV. SM predictions for $B_{s} \rightarrow K K$ decays. Let us put the elements of our analysis together. From the measured value of the branching ratio for $B_{d} \rightarrow K^{0} \bar{K}^{0}$, and choosing a particular value of the direct asymmetry $A_{d i r}^{d 0}$, we get the penguin and tree contributions as explained in III. Then, the bounds in II yield the hadronic parameters in $B_{s} \rightarrow K K$ decays up to small uncertainties. To be more conservative, we actually stretch the bounds in II relating $B_{d}$ and $B_{s}$ hadronic parameters up to $5 \%$ in order to account for well-behaved short-distance $1 / m_{b^{-}}$ suppressed corrections not yet included.

We obtain observables as functions of $A_{d i r}^{d 0}$ in Table In the case of the branching ratios, we have split the error in two parts. The first uncertainty comes from the QCDF estimates of $\Delta_{d}$ and $\bar{\alpha}_{1}$, the theoretical constraints derived in II to relate $B_{d}$ and $B_{s}$ decays and the measurement of $B R^{d 0}$ (this experimental uncertainty dominates the others). The second error stems from (factorisable) $U$-spin breaking terms: $f=0.94 \pm 0.2$ (cf. [2]).

Table \ corresponds only to the solution of the constraints with $\operatorname{Im} P^{d 0}>0$. But $B R^{d 0}, A_{d i r}^{d 0}$ and $\Delta_{d}$ yield two different solutions for $\left(T^{d 0}, P^{d 0}\right)$, and thus for $\left(T^{s \pm}, P^{s \pm}\right)$. Only one solution is physical, whereas the other stems from the non-linear nature of the constraints. We can use flavour-symmetry arguments to lift this ambiguity by exploiting a channel related to $B_{s} \rightarrow K^{+} K^{-}$ through $U$-spin, namely $\bar{B}_{d} \rightarrow \pi^{+} \pi^{-}$[4, 5, 6, 7].

First we apply the method in [5, 7] to the updated average [12] for $\bar{B}_{d} \rightarrow \pi^{+} \pi^{-}: B R=(5.0 \pm 0.4) \times 10^{-6}$, $A_{\text {dir }}=-0.33 \pm 0.11$ and $A_{\text {mix }}=0.49 \pm 0.12$. In this way, we obtain the tree and penguin contributions $\left|T_{\pi \pi}^{d \pm}\right|=(5.48 \pm 0.42) \times 10^{-6},\left|P_{\pi \pi}^{d \pm} / T_{\pi \pi}^{d \pm}\right|=0.13 \pm 0.05$ and $\arg \left(P_{\pi \pi}^{d \pm} / T_{\pi \pi}^{d \pm}\right)=(131 \pm 18)^{\circ}$. Both modulus and phase agree well with their $\bar{B}_{s} \rightarrow K^{+} K^{-}$counterparts as confirmed by the first columns of Table (for $A_{d i r}^{d 0}>0$ ), corresponding to the solution with $\operatorname{Im} P^{d 0}>0$. We get also the $U$-spin breaking parameters $\mathcal{R}_{\mathcal{C}}$ and $\xi$. The last columns give the hadronic parameters for the second solution ( $\operatorname{Im} P^{d 0}<0$ ), to be discarded: $U$-spin would be strongly broken by the phase of the ratio, and we get $A_{d i r}^{s \pm}<0$ contrary to $U$-spin predictions from 


\begin{tabular}{|l||c|c|c||c|c|c|}
\hline & $B R^{s 0} \times 10^{6}$ & $A_{d i r}^{s 0} \times 10^{2}$ & $A_{\text {mix }}^{s 0} \times 10^{2}$ & $B R^{s \pm} \times 10^{6}$ & $A_{\text {dir }}^{s \pm} \times 10^{2}$ & $A_{\text {mix }}^{s \pm} \times 10^{2}$ \\
\hline$A_{\text {dir }}^{d 0}=-0.2$ & $18.4 \pm 6.5 \pm 3.6$ & $0.8 \pm 0.3$ & $-0.3 \pm 0.8$ & $21.9 \pm 7.9 \pm 4.3$ & $24.3 \pm 18.4$ & $24.7 \pm 15.5$ \\
\hline$A_{\text {dir }}^{d 0}=-0.1$ & $18.2 \pm 6.4 \pm 3.6$ & $0.4 \pm 0.3$ & $-0.7 \pm 0.7$ & $19.6 \pm 7.3 \pm 4.2$ & $35.7 \pm 14.4$ & $7.7 \pm 15.7$ \\
\hline$A_{d i r}^{d 0}=0$ & $18.1 \pm 6.3 \pm 3.6$ & $0 \pm 0.3$ & $-0.8 \pm 0.7$ & $17.8 \pm 6.0 \pm 3.7$ & $37.0 \pm 12.3$ & $-9.3 \pm 10.6$ \\
\hline$A_{\text {dir }}^{d 0}=0.1$ & $18.2 \pm 6.4 \pm 3.6$ & $-0.4 \pm 0.3$ & $-0.7 \pm 0.7$ & $16.4 \pm 5.7 \pm 3.3$ & $29.7 \pm 19.9$ & $-26.3 \pm 15.6$ \\
\hline$A_{d i r}^{d 0}=0.2$ & $18.4 \pm 6.5 \pm 3.6$ & $-0.8 \pm 0.3$ & $-0.3 \pm 0.8$ & $15.4 \pm 5.6 \pm 3.1$ & $6.8 \pm 28.9$ & $-40.2 \pm 14.6$ \\
\hline
\end{tabular}

TABLE I: Observables for $\bar{B}_{s} \rightarrow K^{0} \bar{K}^{0}$ and $\bar{B}_{s} \rightarrow K^{+} K^{-}$as functions of the direct asymmetry $A_{d i r}\left(\bar{B}_{d} \rightarrow K^{0} \bar{K}^{0}\right)$ within the SM. We take $\lambda_{u}^{(d)}=0.0038 \cdot e^{-i \gamma}, \lambda_{c}^{(d)}=-0.0094, \lambda_{u}^{(s)}=0.00088 \cdot e^{-i \gamma}, \lambda_{c}^{(s)}=0.04$, and $\gamma=62^{\circ}, \phi_{d}=47^{\circ}, \phi_{s}=-2^{\circ}[13$.

\begin{tabular}{|l|c|c|c||c|c||c|c|c|}
\hline & $\left|T^{s \pm}\right| \times 10^{6}$ & $\left|P^{s \pm} / T^{s \pm}\right|$ & $\arg \left(P^{s \pm} / T^{s \pm}\right)$ & $\mathcal{R}_{\mathcal{C}}$ & $\xi$ & $\left|T^{s \pm}\right|_{\mathrm{rej}} \times 10^{6}$ & $\left|P^{s \pm} / T^{s \pm}\right|_{\mathrm{rej}}$ & $\arg \left(P^{s \pm} / T^{s \pm}\right)_{\mathrm{rej}}$ \\
\hline$A_{\text {dir }}^{d 0}=-0.2$ & $12.7 \pm 2.8$ & $0.09 \pm 0.03$ & $(45 \pm 33)^{\circ}$ & $2.3 \pm 0.7$ & $0.71 \pm 0.24$ & $13.1 \pm 2.9$ & $0.09 \pm 0.03$ & $(-9 \pm 31)^{\circ}$ \\
\hline$A_{\text {dir }}^{d 0}=-0.1$ & $12.1 \pm 2.7$ & $0.10 \pm 0.03$ & $(78 \pm 27)^{\circ}$ & $2.2 \pm 0.7$ & $0.75 \pm 0.27$ & $12.8 \pm 2.9$ & $0.09 \pm 0.03$ & $(-41 \pm 23)^{\circ}$ \\
\hline$A_{\text {dir }}^{d 0}=0$ & $11.5 \pm 2.6$ & $0.10 \pm 0.03$ & $(105 \pm 15)^{\circ}$ & $2.1 \pm 0.6$ & $0.78 \pm 0.31$ & $12.3 \pm 2.8$ & $0.10 \pm 0.03$ & $(-65 \pm 14)^{\circ}$ \\
\hline$A_{\text {dir }}^{d 0}=0.1$ & $11.1 \pm 2.6$ & $0.11 \pm 0.03$ & $(137 \pm 27)^{\circ}$ & $2.0 \pm 0.6$ & $0.82 \pm 0.35$ & $11.8 \pm 2.8$ & $0.10 \pm 0.03$ & $(-90 \pm 28)^{\circ}$ \\
\hline$A_{d i r}^{d 0}=0.2$ & $10.8 \pm 2.6$ & $0.11 \pm 0.03$ & $(180 \pm 10)^{\circ}$ & $2.0 \pm 0.6$ & $0.84 \pm 0.35$ & $11.2 \pm 2.8$ & $0.11 \pm 0.03$ & $(-126 \pm 37)^{\circ}$ \\
\hline
\end{tabular}

TABLE II: Hadronic parameters for $\bar{B}_{s} \rightarrow K^{+} K^{-}$and $U$-spin breaking parameters $\mathcal{R}_{\mathcal{C}}=\left|T^{s \pm} / T_{\pi \pi}^{d \pm}\right|$ and $\xi=$ $\left|P^{s \pm} / T^{s \pm}\right| /\left|P_{\pi \pi}^{d \pm} / T_{\pi \pi}^{d \pm}\right|$ relating $\bar{B}_{s} \rightarrow K^{+} K^{-}$and $\bar{B}_{d} \rightarrow \pi^{+} \pi^{-}$. The last three columns correspond to $\bar{B}_{s} \rightarrow K^{+} K^{-}$hadronic parameters from the second solution for $\bar{B}_{d} \rightarrow K^{0} \bar{K}^{0}$ tree and penguins (rejected due to a large $U$-spin violation).

$\bar{B}_{d} \rightarrow \pi^{+} \pi^{-}$[7, [8]. Thus the two-fold ambiguity can be lifted based on $U$-spin and data on $\bar{B}_{d} \rightarrow \pi^{+} \pi^{-}$.

Table \shows the sign anti-correlation between $A_{m i x}^{s \pm}$ and $A_{d i r}^{d 0}$. $U$-spin arguments applied to $\bar{B}_{d} \rightarrow \pi^{+} \pi^{-}$data indicate $A_{\text {mix }}^{s \pm} \lesssim 0$ [7], and thus $A_{\text {dir }}^{d 0} \gtrsim 0$. Another interesting issue is $B R^{s \pm}$, whose determination is improved compared to the $U$-spin extraction from $\bar{B}_{d} \rightarrow \pi^{+} \pi^{-}$ [5, 7]. Its value is a bit low compared to CDF data [14]:

$$
\begin{aligned}
\left.B R^{s \pm}\right|_{t h} \cdot 10^{6} & =20 \pm 8 \pm 4 \pm 2 & & \text { [our SM result] } \\
\left.B R^{s \pm}\right|_{\exp 1} \cdot 10^{6} & =33 \pm 9 & & {\left[\bar{B}_{d} \rightarrow \pi^{+} K^{-} \text {ratio }\right] } \\
\left.B R^{s \pm}\right|_{\exp 2} \cdot 10^{6} & =42 \pm 15 & & {\left[\bar{B}_{d} \rightarrow \pi^{+} \pi^{-} \text {ratio }\right] }
\end{aligned}
$$

Our SM result is obtained by averaging over the whole range of $A_{d i r}^{d 0}$, although a less conservative restriction to $A_{d i r}^{d 0}>0$ would yield slightly lower central value. The first uncertainty comes from $B R\left(B_{d} \rightarrow K^{0} \bar{K}^{0}\right)$ and $A_{d i r}^{d 0}$, accounting for long-distance $1 / m_{b}$-corrections. The second one comes from the factorisable ratio $f$. When relating $B_{d}$ and $B_{s}$ hadronic parameters in II, the error bars have been stretched to account for $1 / m_{b}$-suppressed contributions that are not enhanced and thus not included in $X_{A, H}$. In addition to this stretching, we give a rough estimate of the same non-enhanced $1 / m_{b}$-suppressed terms through the last error quoted for our SM result. Within these fairly conservative errors, the data suggest a departure from the SM, to be further checked experimentally.

V. Conclusions. We have combined experimental data, flavour symmetries and QCDF to propose sum rules for $B_{d, s} \rightarrow K^{0} \bar{K}^{0}$ observables and to give SM constraints on $B_{s} \rightarrow K \bar{K}$ in a controlled way. We have correlated $B_{s} \rightarrow K \bar{K}$ observables to the direct $B_{d} \rightarrow K^{0} \bar{K}^{0} \mathrm{CP}$ asymmetry and predicted the $U$-spin breaking parameter $\xi$. The main errors on our results in Table \ come from the $U$-spin breaking ratio $f$ (computable on the lattice) and from the experimental value of $B R\left(B_{d} \rightarrow K^{0} \bar{K}^{0}\right)$.

If sizeable NP effects occur, the SM correlations between $B_{d}$ and $B_{s}$ decays exploited here should be broken, leading to departure from our predictions. Indeed we have pointed out a potential conflict between the SM prediction for $B R^{s \pm}$ and experimental data. The ideas developed here could be applied to other non-leptonic $B$-decays, which we leave for future work.

Work supported in part by EU contract EURIDICE (HPRN-CT2002-00311). J.M acknowledges support from RyC program (FPA2002-00748 and PNL2005-41).

[1] M.Beneke, G.Buchalla, M.Neubert and C.T.Sachrajda, Nucl.Phys.B591(2000)313; Nucl.Phys.B606(2001)245

[2] M. Beneke and M. Neubert, Nucl.Phys.B 675 (2003) 333

[3] C.W.Bauer et al., hep-ph/0510241 A.R.Williamson et al., hep-ph/0601214

[4] R. Fleischer, Phys. Lett. B 459 (1999) 306; R. Fleischer and J. Matias, Phys. Rev. D 61 (2000) 074004; Phys. Rev. D 66 (2002) 054009; M. Gronau and J. L. Rosner, Phys. Rev. D 65 (2002) 113008; A. S. Safir, JHEP 0409, 053 (2004); R. Fleischer, J. Phys. G 32 (2006) R71

[5] A. J. Buras et al. Nucl. Phys. B 697 (2004) 133

[6] D. London and J. Matias, Phys.Rev.D 70, 031502 (2004)

[7] D. London, J. Matias and J. Virto, Phys. Rev. D 71, 014024 (2005); S. Baek et al, JHEP 0602, 027 (2006)

[8] M. Beneke, eConf C0304052 (2003) FO001

[9] T. Feldmann and T. Hurth, JHEP 0411, 037 (2004)

[10] R. Fleischer and S. Recksiegel, Eur.Phys.J.C38(2004)251 
[11] B. Aubert et al. [BaBar], Phys. Rev. Lett. 95 (2005) 221801; H. C. Huang [Belle], hep-ex/0205062

[12] B. Aubert et al. [Babar], hep-ex/0501071 hep-ex/0508046 Y. Chao et al. [Belle], Phys. Rev. D 69,
111102 (2004); K. Abe et al. [Belle], hep-ex/0502035

[13] J. Charles et al., Eur. Phys. J. C 41 (2005) 1

[14] D. Tonelli [CDF Collaboration], hep-ex/0512024 